\title{
Divide-and-Evolve: a New Memetic Scheme for Domain-Independent Temporal Planning
}

\author{
Marc Schoenauer $^{1}$, Pierre Savéant ${ }^{2}$, Vincent Vidal $^{3}$ \\ ${ }^{1}$ Projet TAO, INRIA Futurs, LRI, Bt. 490, Université Paris Sud, 91405 Orsay, France \\ 2 Thales Research \& Technology France, RD 128, F-91767 Palaiseau, France \\ ${ }^{3}$ CRIL \& Université d'Artois, rue de l'université - SP16, 62307 Lens, France
}

\begin{abstract}
An original approach, termed Divide-and-Evolve is proposed to hybridize Evolutionary Algorithms (EAs) with Operational Research (OR) methods in the domain of Temporal Planning Problems (TPPs). Whereas standard Memetic Algorithms use local search methods to improve the evolutionary solutions, and thus fail when the local method stops working on the complete problem, the Divide-and-Evolve approach splits the problem at hand into several, hopefully easier, sub-problems, and can thus solve globally problems that are intractable when directly fed into deterministic OR algorithms. But the most prominent advantage of the Divide-and-Evolve approach is that it immediately opens up an avenue for multi-objective optimization, even though the OR method that is used is single-objective. Proof of concept approach on the standard (single-objective) Zeno transportation benchmark is given, and a small original multi-objective benchmark is proposed in the same Zeno framework to assess the multi-objective capabilities of the proposed methodology, a breakthrough in Temporal Planning.
\end{abstract}

\section{Introduction}

Artificial Intelligence Planning is a form of general problem solving task which focuses on problems that map into state models that can be defined by a state space $S$, an initial state $s_{0} \subseteq S$, a set of goal states $S_{G} \subseteq S$, a set of actions $A(s)$ applicable in each state $S$, and a transition function $f(a, s)=s^{\prime}$ with $a \in A(s)$, and $s, s^{\prime} \in S$. A solution to this class of models is a sequence of applicable actions mapping the initial state $s_{0}$ to a goal state that belongs to $S_{G}$.

An important class of problems is covered by Temporal Planning which extends classical planning by adding a duration to actions and by allowing concurrent actions in time [8]. In addition, other metrics are usually needed for real-life problems to qualify a good plan, for instance a cost or a risk criterion. A usual approach is to aggregate the multiple criteria, but this relies on highly problem-dependent features and is not always meaningful. A better solution is to compute the set of optimal non-dominated solutions - the so-called Pareto front.

Because of the high combinatorial complexity and the multi-objective features of Temporal Planning Problems (TPPs), Evolutionary Algorithms are good general-purpose candidate methods. 
However, there has been very few attempts to apply Evolutionary Algorithms to planning problems and, as far as we know, not any to Temporal Planning. Some approaches use a specific representation (e.g. dedicated to the battlefield courses of action [15]). Most of the domain-independent approaches see a plan as a program and rely on Genetic Programming and on the traditional blocksworld domain for experimentation (starting with the Genetic Planner [17]). A more comprehensive state of the art on Genetic Planning can be found in 1] where the authors experimented a variable length chromosome representation. It is important to notice that all those works search the space of (partial) plans.

It is also now well-known that Evolutionary Algorithms (EAs) can rarely efficiently solve Combinatorial Optimization Problems on their own, i.e. without being hybridized, one way or another, with local search ad hoc techniques. The most successful of such hybridizations use Operational Research methods to locally improve any offspring that was born from EA variation operators (crossover and mutation): such algorithms have been termed "Memetic Algorithms" or "Genetic Local Search" 14. Those methods are now the heart of a whole research field, as witnessed by the series of WOMA's (Workshops on Memetic Algorithms) organized every year now, Journal Special Issues and edited books [10.

However, most memetic approaches are based on finding local improvements of candidate solutions proposed by the evolutionary search mechanism using dedicated local search methods that have to tackle the complete problem. In some combinatorial domains such as Temporal Planning, this simply proves to be impossible when reaching some level of complexity.

This paper proposes Divide-and-Evolve, borrowing to the Divide-and-Conquer paradigm for such situations: the problem at hand is sliced into a sequence of problems that are hopefully easier to solve by OR or other local methods. The solution to the original problem is then obtained by a concatenation of the solutions to the different sub-problems.

Next section presents an abstract formulation of the Divide-and-Evolve scheme, and starting from its historical (and pedagogical) root, the TGV paradigm. Generic representation and variation operators are also introduced. Section 3 introduces an actual instantiation of the Divide-and-Evolve scheme to TPPs. The formal framework of TPPs is first introduced, then the TPP-specific issues for the Divide-and-Evolve implementation are presented and discussed. Section 4 is devoted to experiments on the transportation Zeno benchmark for both single and multi-objective cases. The last section opens a discussion highlighting the limitations of the present work and giving hints about on-going and future work.

\section{The Divide-and-Evolve Paradigm}

\subsection{The TGV metaphor}

The Divide-and-Evolve strategy springs from a metaphor on the route planning problem for the French high-speed train (TGV). The original problem consists in computing the shortest route between two points of a geographical landscape 
with strong bounds on the curvature and slope of the trajectory. An evolutionary algorithm was designed [4] based on the fact that the only local search algorithm at hand was a greedy deterministic algorithm that could solve only very simple (i.e. short distance) problems. The evolutionary algorithm looks for a split of the global route into small consecutive segments such that a local search algorithm can easily find a route joining their extremities. Individuals represent sets of intermediate train stations between the station of departure and the terminus. The convergence toward a good solution was obtained with the definition of appropriate variation and selection operators [4. Here, the state space is the surface on which the trajectory of the train is defined.

Generalization Abstracted to Planning, the route is replaced by a sequence of actions and the "stations" become intermediate states of the system. The problem is thus divided into sub-problems and "to be close" becomes "to be easy to solve" by some local algorithm $\mathcal{L}$. The evolutionary algorithm plays the role of an oracle pointing at some imperative states worth to go trough.

\subsection{Representation}

The problem at hand is an abstract AI Planning problem as described in the introduction. The representation used by the evolutionary algorithm is a variable length list of states: an individual is thus defined as $\left(s_{i}\right)_{i \in[1, n]}$, where the length $n$ and all the states $s_{i}$ are unknown and subject to evolution. States $s_{0}$ and $s_{n+1} \equiv s_{G}$ will represent the initial state and the goal of the problem at hand, but will not be encoded in the genotypes. By reference to the original TGV paradigm, each of the states $s_{i}$ of an individual will be called a station.

Requirements The original TGV problem is purely topological with no temporal dimension and reduces to a planning problem with a unique action: moving between two points. The generalization to a given planning domain requires to be able to:

1. define a distance between two different states of the system, so that $d(S, T)$ is somehow related to the difficulty for the local algorithm $\mathcal{L}$ to find a plan mapping the initial state $S$ to the final state $T$;

2. generate a chronological sequence of virtual "stations", i.e. intermediate states of the system, that are close to one another, $s_{i}$ being close to $s_{i+1}$;

3. solve the resulting "easy" problems using the local algorithm $\mathcal{L}$;

4. "glue" the sub-plans into an overall plan of the problem at hand.

\subsection{Variation operators}

This section describes several variation operators that can be defined for the general Divide-and-Evolve approach, independently of the actual domain of application (e.g. TPPs, or the original TGV problem). 
Crossover Crossover operators amounts to exchanging stations between two individuals. Because of the sequential nature of the fitness, it seems a good idea to try to preserve sequences of stations, resulting in straightforward adaptations to variable-length representation of the classical 1- or 2-point crossover operators.

Suppose you are recombining two individuals $\left(s_{i}\right)_{1 \leq ? n}$ and $\left(T_{i}\right)_{1 \leq ? m}$. The $1-$ point crossover amounts to choosing one station in each individual, say $s_{a}$ and $T_{b}$, and exchanging the second part of the lists of stations, obtaining the two offspring $\left(s_{1}, \ldots, s_{a}, T_{m+1}, \ldots T_{b}\right)$ and $\left(T_{1}, \ldots, T_{b}, s_{n+1}, \ldots, s_{n}\right)$ (2-point crossover is easily implemented in a similar way). Note that in both cases, the length of each offspring is likely to differ from those of the parents.

The choice of the crossover points $s_{a}$ and $T_{b}$ can be either uniform (as done in all the work presented here), or distance-based, if some distance is available: pick the first station $s_{a}$ randomly, and choose $T_{b}$ by e.g. a tournament based on the distance with $s_{a}$ (this is on-going work).

Mutation Several mutation operators can be defined. Suppose individual $\left(s_{i}\right)_{1 \leq ? n}$ is being mutated:

- At the individual level, the $A d d$ mutation simply inserts a new station $s_{\text {new }}$ after a given station $\left(s_{a}\right)$, resulting in an $n+1$-long list, $\left(s_{1}, \ldots, s_{a}\right.$, $\left.s_{\text {new }}, s_{a+1}, \ldots, s_{n}\right)$. Its counterpart, the Del mutation, removes a station $s_{a}$ from the list.

Several improvements on the pure uniform choice of $s_{a}$ can be added and are part of on-going work, too: in case the local algorithm fails to successfully join all pairs of successive stations, the last station that was successfully reached by the local algorithm can be preferred for station $s_{a}$ (in both the $A d d$ and Del mutations). If all partial problems are solved, the most difficult one (e.g. in terms of number of backtracks) can be chosen.

- At the station level, the definition of each station can be modified but this is problem-dependent. However, assuming there exists a stationmutation operator $\mu_{S}$, it is easy to define the individual-mutation $M_{\mu_{S}}$ that will simply call $\mu_{S}$ on each station $s_{i}$ with a user-defined probability $p_{\mu_{S}}$. Examples of operators $\mu_{S}$ will be given in section 3 while simple Gaussian mutation of the $(x, y)$ coordinates of a station were used for the original TGV problem [4.

\section{Application to Temporal Planning}

\subsection{Temporal planning problems}

Domain-Independent planners rely on the Planning Domain Definition Language (PDDL) [13, inherited from the STRIPS model [5], to represent a planning problem. In particular, this language is used for a competition ${ }^{1}$ which is held every two years since 1998. The language has been extended for representing

\footnotetext{
1 http://ipc.icaps-conference.org/
} 
Temporal Planning Problems in PDDL2.1 [7]. For the sake of simplicity, the temporal model is often simplified as explained below [18.

A Temporal PDDL Operator is a tuple $o=\langle\operatorname{pre}(o), \operatorname{add}(o), \operatorname{del}(o), \operatorname{dur}(o)\rangle$ where $\operatorname{pre}(o), \operatorname{add}(o)$ and $\operatorname{del}(o)$ are sets of ground atoms that respectively denote the preconditions, add effects and del effects of $o$, and $\operatorname{dur}(o)$ is a rational number that denotes the duration of $o$. The operators in a PDDL input can be described with variables, used in predicates such as (at ?plane ?city).

A Temporal Planning Problem is a tuple $P=\langle A, I, O, G\rangle$, where $A$ is a set of atoms representing all the possible facts in a world situation, $I$ and $G$ are two sets of atoms that respectively denote the initial state and the problem goals, and $O$ is a set of ground PDDL operators.

As is common in Partial Order Causal Link (POCL) Planning 19, two dummy actions are also considered, Start and End with zero durations, the first with an empty precondition and effect $I$; the latter with precondition $G$ and empty effects. Two actions $a$ and $a^{\prime}$ interfere when one deletes a precondition or positive effect of the other. The simple model of time in [16] defines a valid plan as a plan where interfering actions do not overlap in time. In other words, it is assumed that the preconditions need to hold until the end of the action, and that the effects also hold at the end and cannot be deleted during the execution by a concurrent action.

A schedule $P$ is a finite set of actions occurrences $\left\langle a_{i}, t_{i}\right\rangle, i=1, \ldots, n$, where $a_{i}$ is an action and $t_{i}$ is a non-negative integer indicating the starting time of $a_{i}$ (its ending time is $t_{i}+\operatorname{dur}\left(a_{i}\right)$ ). P must include the Start and End actions, the former with time tag 0 . The same action (except for these two) can be executed more than once in $P$ if $a_{i}=a_{j}$ for $i \neq j$. Two action occurrences $a_{i}$ and $a_{j}$ overlap in $P$ if one starts before the other ends; namely if $\left[t_{i}, t_{i}+\operatorname{dur}\left(a_{i}\right)\right] \cap\left[t_{j}, t_{j}+\operatorname{dur}\left(a_{j}\right)\right]$ contains more than one time point.

A schedule $P$ is a valid plan iff interfering actions do not overlap in $P$ and for every action occurrence $\left\langle a_{i}, t_{i}\right\rangle$ in $P$ its preconditions $p \in \operatorname{pre}(a)$ are true at time $t_{i}$. This condition is inductively defined as follows: $p$ is true at time $t=0$ iff $p \in I$, and $p$ is true at time $t>0$ if either $p$ is true at time $t-1$ and no action $a$ in $P$ ending at $t$ deletes $p$, or some action $a^{\prime}$ in $P$ ending at $t$ adds $p$. The makespan of a plan $P$ is the time tag of the End action.

\subsection{CPT: an optimal temporal planner}

An optimal temporal planner computes valid plans with minimum makespan. Even though an optimal planner was not mandatory (as discussed in section 5), we have chosen $C P T$ [18, a freely-available optimal temporal planner, for its temporal dimension and for its constraint-based approach which provide a very useful data structure when it comes to gluing the partial solutions (see section 2.2). Indeed, since in Temporal Planning actions can overlap in time, the simple concatenation of sub-plans, though providing a feasible solution, obviously might produce a plan that is not optimal with respect to the total makespan, even if the sequence of actions is the optimal sequence. However, thanks to the causal links and order constraints maintained by CPT, an improved global plan can 
be obtained by shifting sub-plans as early as possible in a final state of the algorithm.

\subsection{Rationale for using Divide-and-Evolve for Temporal Planning}

The reasons for the failure of standard OR methods addressing TPPs come from the exponential complexity of the number of possible actions when the number of objects involved in the problem increases. It is known for a long time that taking into account the interactions between sub-goals can decrease the complexity of finding a plan, in particular when these sub-goals are independent [12. Moreover, computing an ideal ordering on sub-goals is as difficult as finding a plan (PSPACE-hard), as demonstrated in 11]. The basic idea when using the Divide-and-Evolve approach is that each local sub-plan ("joining" stations $s_{i}$ and $s_{i+1}$ ) should be easier to find than the global plan (joining the station of departure $s_{0}$ and the terminus $\left.s_{n+1}\right)$. This will be now demonstrated on the Zeno transportation benchmark (see http://ipc.icaps-conference.org/).

Table 1 illustrates the decomposition of a relatively difficult problem in the Zeno domain (zeno14 from IPC-3 benchmarks), a transportation problem with 5 planes (plane1 to plane5) and 10 persons (person0 to person9) to travel among 10 cities (city0 to city9).

Analyzing the optimal solution found by CPT-3 it was easy to manually divide the optimal "route" of this solution in the state space into four intermediate stations between the initial state and the goal. It can be seen that very few moves (plane or person) occur between two consecutive stations (the ones in bold in each column of Table 1). Each sub-plan is easily found by CPT, with a maximum of 195 backtracks and 4.34 seconds of search time. It should be noted that most of the time spent by CPT is for pre-processing: this operation is actually repeated each time $\mathrm{CPT}$ is called, but could be factorized at almost no cost.

Note that the final step of the process is the compression of the five sub-plans (see section 2.2): it is here performed in 0.02 seconds without any backtracking, and the overall makespan of the plan is 772 , much less than the sum of the individual makespans of each sub-plan (2051).

To summarize, the recomposed plan, with a makespan of 772 , required a total running time of 254.38 seconds (including only $7.5 \mathrm{~s}$ of pure search) and 228 backtracks altogether, whereas a plan with the optimal makespan of 476 is found by CPT in 4,205 seconds and 606,405 backtracks. Section 5 will discuss this issue.

\subsection{Description of the state space}

Non-temporal states A natural state space for TPPs, as described at the beginning of this section, would be the actual space of all possible time-stamped states of the system. Obviously, the size of such a space is far too big and we simplified it by restricting the stations to non-temporal states. However, even with this simplification, not all "non-temporal" states can be considered in the 
Table 1. State Decomposition of the Zeno14 Instance. (The new location of moved objects appears in bold.)

\begin{tabular}{|c|c|c|c|c|c|c|}
\hline Objects & \begin{tabular}{c|} 
Init \\
$($ station 0$)$
\end{tabular} & Station 1 & Station 2 & Station 3 & Station 4 & $\begin{array}{c}\text { Goal } \\
\text { (station 5) }\end{array}$ \\
\hline plane 1 & city 5 & city 6 & city 6 & city 6 & city 6 & city 6 \\
\hline plane 2 & city 2 & city 2 & city 3 & city 3 & city 3 & city 3 \\
\hline plane 3 & city 4 & city 4 & city 4 & city 9 & city 9 & city 9 \\
\hline plane 4 & city 8 & city 8 & city 8 & city 8 & city 5 & city 5 \\
\hline plane 5 & city 9 & city 9 & city 9 & city 9 & city 9 & city 8 \\
\hline person 1 & city 9 & city 9 & city 9 & city 9 & city 9 & city 9 \\
\hline person 2 & city 1 & city 1 & city 1 & city 1 & city 1 & city 8 \\
\hline person 3 & city 0 & city 0 & city 2 & city 2 & city 2 & city 2 \\
\hline person 4 & city 9 & city 9 & city 9 & city 7 & city 7 & city 7 \\
\hline person 5 & city 6 & city 6 & city 6 & city 6 & city 6 & city 1 \\
\hline person 6 & city 0 & city 6 & city 6 & city 6 & city 6 & city 6 \\
\hline person 7 & city 7 & city 7 & city 7 & city 7 & city 5 & city 5 \\
\hline person 8 & city 6 & city 6 & city 6 & city 6 & city 6 & city 1 \\
\hline person 9 & city 4 & city 4 & city 4 & city 4 & city 5 & city 5 \\
\hline person 0 & city 7 & city 7 & city 7 & city 9 & city 9 & city 9 \\
\hline \multicolumn{2}{|c|}{ Makespan } & 350 & 350 & 280 & 549 & 522 \\
\hline \multicolumn{2}{|c|}{ Backtracks } & 1 & 0 & 0 & 195 & 32 \\
\hline \multicolumn{2}{|c|}{ Search time } & 0.89 & 0.13 & 0.52 & 4.34 & 1.64 \\
\hline \multicolumn{2}{|c|}{ Total time } & 49.10 & 49.65 & 49.78 & 54.00 & 51.83 \\
\hline & & \multicolumn{2}{|c|}{ Compression } & & \multicolumn{2}{|c|}{ Global Search } \\
\hline \multicolumn{2}{|c|}{ Makespan } & \multicolumn{2}{|c|}{772} & & \multicolumn{2}{|c|}{476} \\
\hline \multicolumn{2}{|c|}{ Backtracks } & \multicolumn{2}{|c|}{0} & & \multicolumn{2}{|c|}{606,405} \\
\hline \multicolumn{2}{|c|}{ Search time } & \multicolumn{2}{|c|}{0.01} & & \multicolumn{2}{|c|}{$4,155.41$} \\
\hline \multicolumn{2}{|c|}{ Total time } & \multicolumn{2}{|c|}{$0.02($ total : 254.38$)$} & & \multicolumn{2}{|c|}{$4,205.40$} \\
\hline
\end{tabular}

description of the "stations".

Limiting the possible states First, the space of all possible states grows exponentially with the size of the problem. Second, not all states are consistent w.r.t. the planning domain. For instance, an object cannot be located at two places at the same time in a transportation problem - and inferring such state invariants is feasible but not trivial 6 . Note also that determining plan existence from a propositional STRIPS description has been proved to be PSPACE-complete 2].

A possible way to overcome this difficulty would be to rely on the local algorithm to (rapidly) check the consistency of a given situation, and to penalize unreachable stations. However, this would clearly be a waste of computational resources.

On the other hand, introducing domain knowledge into EAs has been known for long as the royal road toward success in Evolutionary Computation 9. Hence, it seems a more promising approach to add state invariants to the description of the state space in order to remove the inconsistent states as much as possible. The good thing is that it is not necessary to remove all inconsistent states 
since, in any case, the local algorithm is there to help the EA to spot them inconsistent stations will be given poor fitness, and will not survive next selection steps. In particular, only state invariants involving a single predicate have been implemented in the present work.

\subsection{Representation of stations}

It was hence decided to describe the stations using only the predicates that are present in the goal of the overall problem, and to maintain the state invariants based on the semantics of the problem.

A good example is given in Table 1 the goal of this benchmark instance is to move the persons and planes in cities listed in the last column. No other predicate than the corresponding (at objectN cityM) predicates is present in the goal. Through a user-supplied file, the algorithm is told that only the at predicates will be used to represent the stations, with the syntactic restrictions that within a given station, the first argument of an at predicate can appear only once (at is said to be exclusive with respect to its first argument). The state space that will be explored by the algorithm thus amounts to a vector of 15 fluents (instantiated predicates) denoting that an item is located in a city (a column of table 11). In addition, the actual implementation of a station includes the possibility to "remove" (in fact, comment out) a predicate of the list: the corresponding object will not move during this sub-plan.

Distance The distance between two stations should reflect the difficulty for the local algorithm to find a plan joining them. At the moment, a purely syntactic domain-independent distance is used: the number of different predicates not yet reached. The difficulty can then be estimated by the number of backtracks needed by the local algorithm. It is reasonable to assume that indeed most local problems where only a few predicates need to be changed from the initial state to the goal will be easy for the local algorithm - though this is certainly not true in all cases.

\subsection{Representation-specific operators}

Initialization First, the number of stations is chosen uniformly in a usersupplied interval. The user also enters a maximal distance $d_{\max }$ between stations. A matrix is then built, similar to the top lines of table 1 each line corresponds to one of the goal predicates, each column is a station. Only the first and last columns (corresponding to initial state and goal) are filled with values. A number of "moves" is then randomly added in the matrix, at most $d_{\text {max }}$ per column, and at least one per line. Additional moves are then added according to another user-supplied parameter, and without exceeding the $d_{\max }$ limit per column. The matrix is then filled with values, starting from both ends (init and goal), constrained column-wise by the state invariants. A final sweep on all predicates comments out some of the predicates with a given probability. 
Station mutation Thanks to the simplified representation of the states (a vector of fluents with a set of state invariants), it is straightforward to modify one station randomly: with a given probability, a new value for the non-exclusive arguments is chosen among the possible values respecting all constraints (including the distance constraints with previous and next stations). In addition, each predicate might be commented out from the station with a given probability, like in the initialization phase.

\section{First Experiments}

\subsection{Single objective optimization}

Our main playground to validate the Divide-and-Evolve approach is that of transportation problems, and started with the zeno domain as described in section 3.3 As can be seen in table 1 the description of the stations in zeno domain involves a single predicate, at, with two arguments. It is exclusive w.r.t. its first argument. Three instances have been tried, called zeno10, zeno12 and zeno14, from the simplest to the hardest.

Algorithmic settings The EA that was used for the first implementation of the Divide-and-Evolve paradigm use standard algorithmic settings at the population level: a $(10,70)-E S$ evolution engine (10 parents give birth to 70 children, and the best 10 among the children become the next parents), the children are created using 25\% 1-point crossover (see section 2.3) and 75\% mutation (individual level), out of which $25 \%$ are the $A d d$ (resp. Del) generic mutations (section 2.3). The remaining $50 \%$ of the mutations call the problem-specific station mutation. Within a station mutation, a predicate is randomly changed in $75 \%$ of the cases and a predicate is removed (resp. restored) in each of the remaining $12.5 \%$ cases. (see section 3.6). Initialization is performed using initial size in $[2,10]$, maximum distance of 3 and probability to comment out a predicate is set to 0.1 . Note that no lengthy parameter tuning was performed for those proof-of-concept experiments, and the above values were decided based upon a very limited set of initial experiments.

The fitness The target objective is here the total makespan of a plan - assuming that a global plan can be found, i.e. that all problems $\left(s_{i}, s_{i+1}\right)$ can be solved by the local algorithm. In case one of the local problems could not be solved, the individual is declared infeasible and is penalized in such a way that all unfeasible individuals were worse than any feasible one. Moreover, this penalty is proportional to the number of remaining stations after the failure, in order to provide a nice slope of the fitness landscape toward feasibility. For feasible individuals, an average of the total makespan and the sum of the makespans of all partial problems is used: when only the total makespan is used, some individuals start bloating, without much consequence on the total makespan thanks to the final compression that is performed by $\mathrm{CPT}$, but nevertheless slowing down the whole 
run because of all the useless repeated calls to CPT.

Preliminary results The simple zeno10 (resp. zeno12) instance can be solved very easily by CPT-2 alone, in less than $2 \mathrm{~s}$ (resp. 125s), finding the optimal plans with makespan 453 (resp. 549) using 154 (resp. 27560) backtracks.

For zeno10, all runs found the optimal solution in the very first generations (i.e. the initialization procedure always produced a feasible individual that CPT could compress to the optimal makespan. For zeno12, all runs found a suboptimal solution with makespan between 789 and 1222. Note that this final solution was found after 3 to 5 generations, the algorithm being stuck to this solution thereafter. The CPU time needed for 10 generations is around 5 hours.

A more interesting case is that of zeno14. First of all, it is worth mentioning that the present Divide-and-Evolve EA uses as local algorithm CPT version 2, and this version of CPT was unable to find a solution to zeno14: the results given in table 1 have been obtained using the (yet experimental and not usable from within the EA) new version of CPT. But whereas it proved unable to solve the full problem, CPT-2 could nevertheless be used to solve the hopefully small instances of zeno14 domain that were generated by the Divide-and-Evolve approach - though taking a huge amount of CPU time for that. Setting a limit on the number of backtracks allowed for CPT was also mandatory to force CPT not to explore the too complex cases that would have resulted in a never-returning call.

However, a feasible individual was found in each of the only 2 runs we could run - one generation (70 evaluations) taking more than 10 hours. In the first run, a feasible individual was found in the initial population, with makespan 1958 , and the best solution had a makespan of 773 . In the other run, the first feasible solution was found at generation 3 - but the algorithm never improved on that first feasible individual (makespan 1356).

Though disappointing with respect to the overall performances of the algorithm, those results nevertheless witness for the fact that the Divide-and-Evolve approach can indeed solve a problem that could not be solved by CPT alone (remember that the version of CPT that was used in all experiments is by far less efficient than the one used to solve zeno14 in section 3.3. and was not able to solve zeno14 at all.

\subsection{A multi-objective problem}

Problem description In order to test the feasibility of the multi-objective approach based on the Divide-and-Evolve paradigm, we extended the zeno benchmark with an additional criterion, that can be interpreted either as a cost, or as a risk: in the former case, this additional objective is an additive measure, whereas in the latter case (risk) the aggregation function is the max operator.

The problem instance is shown in Figure 1 the only available routes between cities are displayed as edges, only one transportation method is available (plane), and the duration of the transport is shown on the corresponding edge. Risks (or costs) are attached to the cities (i.e., concern any transportation that either 
lands or takes off from that city). In the initial state, the 3 persons and the 2 planes are in City 0, and the goal is to transport them into City 4.

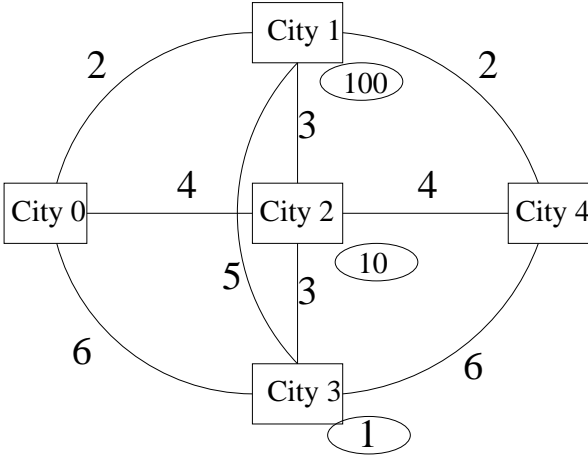

a) The instance: Durations are attached to edges, costs/risks are attached to cities (in gray circles).

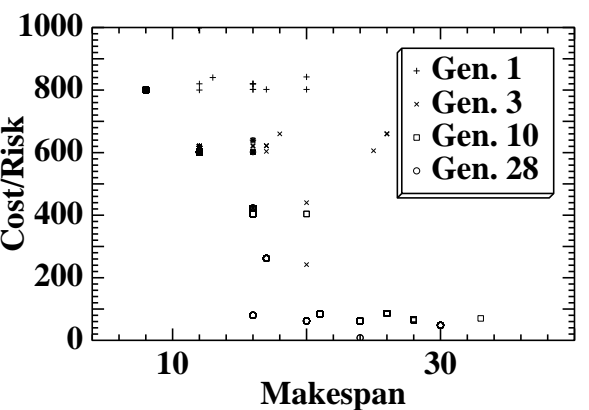

b) The population at different generations for a successful run on the cost (additive) instance of the zeno miniproblem of Figure 1-a.

Fig. 1. The multi-objective Zeno benchmark.

As can be easily computed (though there is a little trick here), there are 3 remarkable Pareto-optimal solutions, corresponding to traversing only one of the 3 middle cities. Going through City 1 is fast, but risky (costly), whereas going through City 3 is slow and safe and cheap.

When all persons go through respectively City 1, City 2 and City 3, the corresponding values of the makespans and costs in the additive case are ( 8 , $800),(16,80)$ and $(24,8)$, whereas they are, in the max case, $(8,100)$, $(16,10)$ and $(24,1)$.

Problem complexity It is easy to compute the number of possible virtual stations: each one of the 3 persons can be in one of the 5 cities, or not mentioned (absent predicate). Hence there are $3^{6}=729$ possible combinations, and $729^{n}$ possible lists of length $n$. So even when $n$ is limited to 6 , the size of the search space is approx. $10^{17} \ldots$

The algorithm The EA is based on the standard NSGA-II multi-objective EA 3]: standard tournament selection of size 2 and deterministic replacement among parents + offspring, both based on the Pareto ranking and crowding distance selection; a population size of 100 evolves during 30 generations. All other parameters were those used for the single objective case.

Fitnesses The problem has two objectives: one is the the total makespan (as in the single-objective case), the other is either the risk (aggregated using the max 
operator) or the cost (an additive objective). Because the global risk only takes 3 values, there is no way to have any useful gradient information when used as fitness in the max case. However, even in the additive case, the same arguments than for the makespan apply (section 4.1), and hence, in all cases, the second objective is the sum of the overall risk/cost and the average (not the sum) of the values for all partial problems - excluding from this average those partial problems that have a null makespan (when the goal is already included in the initial state).

Results For the additive (cost) case, the most difficult Pareto optimum (going through city 3 only) was found 4 times out of 11 runs. However, the 2 other remarkable Pareto optima, as well as several other points in the Pareto front were also repeatedly found by all runs. Figure 1-b shows different snapshots of the population at different stages of the evolution for a typical successful run: at first ('+'), all individuals have a high cost (above 800); At generation 3 $(' \times)$, there exist individuals in the population that have cost less than 600; At generation 10 (squares), many points have a cost less than 100. But the optimal $(24,8)$ solution is only found at generation 28 (circles).

The problem in the risk context (the max case) proved to be, as expected, slightly more difficult. All three Pareto optima (there exist no other point of the true Pareto front in the max case) were found only in 2 runs out of 11 . However, all runs found both the two other Pareto optima, as well as the slightly suboptimal solutions that goes only through city 3 but did not find the little trick mentioned earlier, resulting in a $(36,1)$ solution.

In both cases, those results clearly validate the Divide-and-Evolve approach for multi-objective TPPs - remember that CPT has no knowledge of the risk/cost in its optimization procedure - it only aggregates the values a posteriori, after having computed its optimal plan based on the makespan only - hence the difficulty to find the 3rd Pareto optimum going only through city3.

\section{Discussion and Further Work}

A primary concern is the existence of a decomposition for any plan with optimal makespan. Because of the restriction of the representation to the predicates that are in the goal, some states become impossible to describe. If one of these states is mandatory for all optimal plans, the evolutionary algorithm is unable to find the optimal solution. In the zeno14 benchmark detailed in section 3.3 for instance, one can see from the optimal solution that the in predicate should be taken into account when splitting the optimal solution, in order to be able to link a specific person to a specific plane. The main difficulty, however, is to add the corresponding state invariant between at and in (a person is either at a location or in a plane). Future work will include state invariants involving pairs of predicates, to cope with such cases. Along the same line, we will investigate whether it might be possible to automatically infer some state invariants from the data structures maintained by CPT. 
It is clear from the somehow disappointing results presented in section 4.1 that the search capabilities of the proposed algorithm should be improved. But there is a lot of space for improvements. First, and most immediate, the variation operators could use some domain knowledge, as proposed in section 2.3 - even if this departs from "pure" evolutionary blind search. Also, all parameters of the algorithm will be carefully fine-tuned.

Of course the Divide-and-Evolve scheme has to be experimented on more examples. The International Planning Competition provides many instances in several domains that are good candidates. Preliminary results on the driver problem showed very similar results that those reported here on the zeno domain. But other domains, such as the depot domain, or many real-world domains, involve (at least) 2 predicates in their goal descriptions (e.g., in and on for depot). It is hence necessary to increase the range of allowed expressions in the description of individuals.

Other improvements will result from the move to the new version of CPT, entirely rewritten in C. It will be possible to call CPT from within the EA, and hence to perform all grounding, pre-processing and CSP representation only once: at the moment, CPT is launched anew for each partial computation, and a quick look at table 1 shows that on zeno14 problem, for instance, the run-time per individual will decrease from 250 to 55 seconds. Though this will not per se improve the quality of the results, it will allow us to tackle more complex problems than even zeno14. Along the same lines, other planners, in particular sub-optimal planners, will also be tried in lieu of CPT, as maybe the Divideand-Evolve approach could find optimal results using sub-optimal planners (as done in some sense in the multi-objective case, see section 4.2).

A last but important remark about the results is that, at least in the single objective case, the best solution found by the algorithm was always found in the very early generations of the runs: it could be that the simple splits of the problem into smaller sub-problems that are done during the initialization are the main reasons for the results. Detailed investigations will show whether or not an Evolutionary Algorithm is useful in that context!

Nevertheless, we do believe that using Evolutionary Computation is mandatory in order to solve multi-objective optimization problems, as witnessed by the

results of section 4.2 that are, to the best of our knowledge, the first ever results of Pareto optimization for TPPs.

\section{References}

1. A. H. Brie and P. Morignot. Genetic Planning Using Variable Length Chromosomes. In 15th Intl Conf. on Automated Planning and Scheduling, 2005.

2. T. Bylander. The Computational Complexity of Propositional STRIPS planning. Artificial Intelligence, 69(1-2):165-204, 1994.

3. K. Deb, S. Agrawal, A. Pratab, and T. Meyarivan. A Fast Elitist Non-Dominated Sorting Genetic Algorithm for Multi-Objective Optimization. In M. Schoenauer et al., editor, PPSN'2000, pages 849-858. Springer-Verlag, LNCS 1917, 2000. 
4. C. Desquilbet. Détermination de trajets optimaux par algorithmes génétiques. Rapport de stage d'option B2 de l'Ecole Polytechnique. Palaiseau, France, Juin 1992. Advisor: Marc Schoenauer. In French.

5. R. Fikes and N. Nilsson. STRIPS: A New Approach to the Application of Theorem Proving to Problem Solving. Artificial Intelligence, 1:27-120, 1971.

6. M. Fox and D. Long. The Automatic Inference of State Invariants in TIM. Journal of Artificial Intelligence Research, 9:367-421, 1998.

7. M. Fox and D. Long. PDDL2.1: An Extension to PDDL for Expressing Temporal Planning Domains. Journal of Artificial Intelligence Research, 20:61-124, 2003.

8. H. Geffner. Perspectives on Artificial Intelligence Planning. In Proc. AAAI-2002, pages 1013-1023, 2002.

9. J. J. Grefenstette. Incorporating Problem Specific Knowledge in Genetic Algorithms. In Davis L., editor, Genetic Algorithms and Simulated Annealing, pages 42-60. Morgan Kaufmann, 1987.

10. W.E. Hart, N. Krasnogor, and J.E. Smith, editors. Recent Advances in Memetic Algorithms. Studies in Fuzziness and Soft Computing, Vol. 166. Springer Verlag, 2005.

11. J. Koehler and J. Hoffmann. On Reasonable and Forced Goal Orderings and their Use in an Agenda-Driven Planning Algorithm. JAIR, 12:338-386, 2000.

12. R. Korf. Planning as Search: A Quantitative Approach. Artificial Intelligence, 33:65-88, 1987.

13. D. McDermott. PDDL - The Planning Domain Definition language. At http://ftp.cs.yale.edu/pub/mcdermott, 1998.

14. P. Merz and B. Freisleben. Fitness Landscapes and Memetic Algorithm Design. In David Corne, Marco Dorigo, and Fred Glover, editors, New Ideas in Optimization, pages 245-260. McGraw-Hill, London, 1999.

15. J.L. Schlabach, C.C. Hayes, and D.E. Goldberg. FOX-GA: A Genetic Algorithm for Generating and Analyzing Battlefield Courses of Action. Evolutionary Computation, 7(1):45-68, 1999.

16. D. Smith and D. S. Weld. Temporal Planning with Mutual Exclusion Reasoning. In Proceedings of IJCAI-99, pages 326-337, 1999.

17. L. Spector. Genetic Programming and AI Planning Systems. In Proc. AAAI 94, pages 1329-1334. AAAI/MIT Press, 1994.

18. V. Vidal and H. Geffner. Branching and Pruning: An Optimal Temporal POCL Planner based on Constraint Programming. In Proceedings of AAAI-2004, pages 570-577, 2004.

19. D. S. Weld. An Introduction to Least Commitment Planning. AI Magazine, 15(4):27-61, 1994. 